\title{
Revisão de critérios na admissão de pessoas com restrições médicas no Brasil
}

\author{
Review of criteria adopted for hiring procedures \\ of workers with medical restrictions in Brazil
}

\author{
James César da Silva Jabour Arbex'
}

\begin{abstract}
RESUMO | Contexto: A inclusão de pessoas com restrições médicas no mercado de trabalho é tema de fundamental importância e grande desafio. No Brasil, ínfima parcela dessas pessoas acaba sendo atendida, por obrigação ao cumprimento do Decreto-lei 3.298 de dezembro de 1999, que trata exclusivamente de deficiências. Contudo, um grande grupo de pessoas que não se enquadram nos critérios de deficiências sugeridos se encontra desamparado pela legislação em vigor. Objetivo: Discutir a inclusão de pessoas com restrições médicas no Brasil. Métodos: Estudo da literatura médica e análise dos atuais critérios adotados pelo Ministério do Trabalho e Emprego para admissão de pessoas com restrições médicas. Resultados: O crescimento das admissões de pessoas com deficiência é significativo, apesar de só há pouco tempo o assunto ser tratado como relevante. A fiscalização contínua e efetiva terá papel fundamental no resultado final, mas mostra também que a situação atual não atende a realidade das pessoas com restrições médicas ao trabalho. Conclusão: Embora haja legislação que aborde a contratação de deficientes no Brasil, grande número de pessoas com restrições médicas se encontram desamparadas ou em benefícios sociais quando poderiam e deveriam estar trabalhando.

Palavras-chave I mercado de trabalho; pessoas com deficiência; avaliação da capacidade de trabalho.
\end{abstract}

ABSTRACT | Context: The inclusion of people with medical restrictions in the job market is a crucially important theme and a great challenge. In Brazil, a tiny fraction of these people end up being hired as an obligation to comply with the Decree-law 3,298 of December 1999 that exclusively concerns the disabled. However, a large group of people who do not fit the listed criteria find themselves without assistance from the law. Objective: To discuss the inclusion of people with medical restrictions in Brazil. Methods: Study of medical literature and analysis of current criteria adopted by the Ministry of Labour and Employment for the admission of people with medical restrictions. Results: The increase in admissions of people with disabilities is significant, despite this subject only begins to be treated as relevant recently. A continuous and effective monitoring shall have a fundamental role in the final result, but it shows that the present situation does not meet the needs of people with medical restrictions to work. Conclusion: In spite of having legislation that addresses the employment of disabled people in Brazil, a large number of people with medical restrictions are helpless or receiving social benefits when they can and should be working.

Keywords I job market; disabled persons; work capacity evaluation. 


\section{INTRODUÇÃO}

A Declaração Universal dos Direitos Humanos, produzida pela Assembleia Geral das Nações Unidas, em 10 de dezembro de 1948, diz em seu artigo 23: "toda pessoa tem direito ao trabalho, à livre escolha do trabalho, a condições equitativas e satisfatórias de trabalho e à proteção contra o desemprego" ${ }^{\text {" }}$.

Considerando que o relatório da Organização Mundial da Saúde de 2011 estima que $15 \%$ da população do mundo todo - isto é, 1 bilhão de pessoas — viva com algum tipo de deficiência e que aproximadamente 90 milhões desse total se encontram na região ibero-americana, nada mais importante ${ }^{2}$.

Se for levado em consideração que o mesmo relatório estima que a deficiência seja maior nos países de menor renda; que a população feminina apresente uma maior prevalência; que em muitos desses países essas pessoas vivam em condições de extrema pobreza, com acesso limitado a serviços públicos, como educação e saúde, e em situação de marginalização social; que essa situação determine a elevada taxa de desemprego; e considerando que $80 \%$ dessas pessoas em idade de trabalho estão desempregadas por falta de acessibilidade, o tema de relevante passa a urgente ${ }^{2}$.

Ocorre que, como pode ser visto nos números citados, o problema está bem longe de ser resolvido e, para piorar, acabou criando outro. Toda lei, mesmo correta, com o passar do tempo pode e deve ser melhorada e adequada à sua sociedade; e é isso que este trabalho procura abordar.

O presente artigo tem como objetivo realizar, por meio de atualização da literatura, uma revisão dos critérios vigentes na admissão de pessoas com restrições.

Será fundamental o debate em todas as esferas representadas pelo empresariado, governo, sindicatos de classe e organizações não governamentais (ONGs), a fim de se chegar a um denominador comum para a inclusão de pessoas com restrições médicas de maneira integral.

\section{MÉTODOS}

Foi realizada uma revisão bibliográfica com pesquisa nos bancos de dados do Ministério do Trabalho e Emprego (MTE), do censo do Instituto Brasileiro de Geografia e Estatística (IBGE) de 2010, livros técnicos (autores como Hudson Couto, Primo A. Brandmiller), artigos de revistas científicas (Associação Nacional de Medicina do Trabalho - ANAMT), leituras de decretos e legislações vigentes que tratam da inclusão de deficientes (Lei 8.213 de 24 de julho de 1991, Decreto-lei 5.296 de 2 de Dezembro de 2004, Instrução Normativa 98 de 15 de Agosto de 2012), além de pesquisas em sites como Sociedades Brasileiras de Endocrinologia, Cardiologia, Neurologia, Nefrologia, entre outros citados nas Referências.

\section{RESULTADOS}

Segundo dados do IBGE, o primeiro levantamento censitário brasileiro que tratou do assunto "deficiência" data de 1872. De um censo para outro, houve mudanças nos conceitos ou formulação das perguntas, o que não permite a comparação direta entre esses levantamentos.

No censo de 2010, foram investigadas as deficiências visual, auditiva, motora e mental/intelectual. Além disso, para as três primeiras, também foi verificado o grau de severidade. Os resultados revelam que, no Brasil, $23,9 \%$ da população tinha algum tipo de deficiência, o que significa cerca de 45,6 milhões de pessoas (Tabela 1).

\section{DEFICIÊNCIA}

Foi pesquisada a existência dos seguintes tipos de deficiência permanente: visual, auditiva e motora, de acordo com o seu grau de severidade, e, também, mental ou intelectual. $\mathrm{O}$ foco será em visual e auditiva, que serão aqui discutidas.

Como não existe uma correlação entre os dados censitários acima e a legislação de deficiência brasileira, entende-se que apenas uma pequena parte dos que se apresentam como "com grande dificuldade" estariam contemplados nos critérios de deficiência, mostrando, assim, o imenso número de pessoas que não estão enquadradas.

Além das acima citadas, existem diversas situações médicas que, no entendimento deste autor, causam restrições ao trabalho, como, por exemplo: hipertensão arterial importante ou cardiopatia; diabetes mellitus tipo I; epilepsia; câncer controlado em acompanhamento; obesidade mórbida; insuficiência renal crônica ou hepatopatia grave; tuberculose; síndrome da imunodeficiência adquirida (SIDA — em inglês, acquired immunodeficiency syndrome, AIDS); espondilite anquilosante; surdez unilateral e dificuldade auditiva; e patologias psiquiátricas. 
Tabela 1. População residente, por tipo de deficiência, segundo a situação do domicílio e os grupos de idade. Brasil, 2010.

\begin{tabular}{|c|c|c|c|c|c|c|c|c|}
\hline \multirow{4}{*}{$\begin{array}{l}\text { Situação do } \\
\text { domicílio e } \\
\text { grupos de idade }\end{array}$} & \multicolumn{8}{|c|}{ População residente } \\
\hline & \multirow[b]{3}{*}{ Total (1) (2) } & \multicolumn{7}{|c|}{ Tipo de deficiência } \\
\hline & & \multirow{2}{*}{$\begin{array}{l}\text { Pelo menos } \\
\text { uma das } \\
\text { deficiências } \\
\text { investigadas } \\
\text { (1) }\end{array}$} & \multicolumn{3}{|c|}{ Visual } & \multicolumn{3}{|c|}{ Auditiva } \\
\hline & & & $\begin{array}{l}\text { Não } \\
\text { consegue } \\
\text { de modo } \\
\text { algum }\end{array}$ & $\begin{array}{c}\text { Grande } \\
\text { dificuldade }\end{array}$ & $\begin{array}{c}\text { Alguma } \\
\text { dificuldade }\end{array}$ & $\begin{array}{l}\text { Não } \\
\text { consegue } \\
\text { de modo } \\
\text { algum }\end{array}$ & $\begin{array}{c}\text { Grande } \\
\text { dificuldade }\end{array}$ & $\begin{array}{c}\text { Alguma } \\
\text { dificuldade }\end{array}$ \\
\hline Total (anos) & 190755799 & 45606048 & 506377 & 6056533 & 29211482 & 344206 & 1798967 & 7574145 \\
\hline O a 4 & 13806733 & 385303 & 20935 & 24707 & 122581 & 13593 & 10996 & 54453 \\
\hline 5 a 9 & 14967767 & 1147368 & 21407 & 97719 & 670799 & 16494 & 31976 & 184925 \\
\hline 10 a 14 & 17167135 & 1926730 & 24058 & 175176 & 1286971 & 22379 & 45914 & 235471 \\
\hline 15 a 19 & 16986788 & 2017529 & 24457 & 195493 & 1357295 & 24836 & 44564 & 219824 \\
\hline 15 a 17 & 10353865 & 1218607 & 14475 & 117495 & 821618 & 14373 & 27442 & 133384 \\
\hline 18 e 19 & 6632922 & 798921 & 9981 & 77998 & 535677 & 10463 & 17122 & 86439 \\
\hline 20 a 24 & 17240864 & 2215799 & 29808 & 210571 & 1473070 & 30591 & 48795 & 255109 \\
\hline 25 a 29 & 17102917 & 2376938 & 35860 & 232451 & 1540445 & 31146 & 53492 & 288966 \\
\hline 30 a 34 & 15744616 & 2447685 & 34986 & 235409 & 1523122 & 30538 & 63894 & 325833 \\
\hline 35 а 39 & 13888191 & 2590841 & 32346 & 258624 & 1604547 & 26753 & 70325 & 362784 \\
\hline 40 a 44 & 13008496 & 3797150 & 31166 & 438135 & 2642127 & 23843 & 85537 & 444978 \\
\hline 45 a 49 & 11834647 & 4763491 & 31233 & 617095 & 3481074 & 18724 & 97630 & 529426 \\
\hline 50 a 54 & 10134322 & 4705129 & 28184 & 655232 & 3337231 & 17408 & 119958 & 625726 \\
\hline 55 a 59 & 8284433 & 4170185 & 28068 & 605386 & 2819567 & 15520 & 130589 & 668086 \\
\hline 60 a 64 & 6503287 & 3524275 & 25855 & 527765 & 2258647 & 13267 & 141022 & 686776 \\
\hline 65 a 69 & 4852789 & 2894694 & 24058 & 458022 & 1748246 & 11925 & 147136 & 678305 \\
\hline 70 a 74 & 3744738 & 2451628 & 23652 & 426442 & 1381745 & 10571 & 164179 & 669689 \\
\hline 75 а 79 & 2570686 & 1839631 & 24466 & 353344 & 947089 & 10000 & 169752 & 561265 \\
\hline 80 ou mais & 2917391 & 2351671 & 65840 & 544962 & 1016924 & 26618 & 373207 & 782529 \\
\hline Urbana (anos) & 160934649 & 38473702 & 438481 & 5033221 & 24701413 & 289561 & 1489770 & 6316136 \\
\hline O a 4 & 11311974 & 311301 & 17566 & 19927 & 100529 & 11081 & 8722 & 43407 \\
\hline 5 a 9 & 12136881 & 954356 & 18216 & 81579 & 570932 & 13449 & 25044 & 148527 \\
\hline 10 a 14 & 13959711 & 1607756 & 21004 & 146524 & 1092336 & 18017 & 35675 & 188323 \\
\hline 15 a 19 & 14035653 & 1713004 & 20956 & 167649 & 1172093 & 20582 & 35507 & 179242 \\
\hline 15 a 17 & 8486650 & 1027375 & 12369 & 100115 & 704124 & 11823 & 21792 & 107361 \\
\hline 18 e 19 & 5549002 & 685628 & 8587 & 67535 & 467969 & 8759 & 13715 & 71881 \\
\hline 20 a 24 & 14714074 & 1927620 & 26160 & 184182 & 1303764 & 25758 & 40305 & 214042 \\
\hline 25 a 29 & 14773215 & 2073755 & 31696 & 203609 & 1363595 & 26569 & 44659 & 245150 \\
\hline 30 a 34 & 13613351 & 2117620 & 31193 & 202569 & 1332567 & 26239 & 53118 & 275911 \\
\hline
\end{tabular}


Tabela 1. Continuação.

\begin{tabular}{|c|c|c|c|c|c|c|c|c|}
\hline \multirow{4}{*}{$\begin{array}{l}\text { Situação do } \\
\text { domicílio e } \\
\text { grupos de idade }\end{array}$} & \multicolumn{8}{|c|}{ População residente } \\
\hline & \multirow[b]{3}{*}{ Total (1) (2) } & \multirow{3}{*}{$\begin{array}{c}\text { Pelo menos } \\
\text { uma das } \\
\text { deficiências } \\
\text { investigadas } \\
\text { (1) }\end{array}$} & \multirow{2}{*}{\multicolumn{3}{|c|}{ Tipo de deficiênc }} & \multirow{2}{*}{\multicolumn{3}{|c|}{ Auditiva }} \\
\hline & & & & & & & & \\
\hline & & & $\begin{array}{l}\text { Não } \\
\text { consegue } \\
\text { de modo } \\
\text { algum }\end{array}$ & $\begin{array}{c}\text { Grande } \\
\text { dificuldade }\end{array}$ & $\begin{array}{c}\text { Alguma } \\
\text { dificuldade }\end{array}$ & $\begin{array}{l}\text { Não } \\
\text { consegue } \\
\text { de modo } \\
\text { algum }\end{array}$ & $\begin{array}{c}\text { Grande } \\
\text { dificuldade }\end{array}$ & $\begin{array}{l}\text { Alguma } \\
\text { dificuldade }\end{array}$ \\
\hline 35 а 39 & 11970596 & 2215056 & 28566 & 220152 & 1379536 & 22190 & 58849 & 307721 \\
\hline 40 a 44 & 11180729 & 3208708 & 27263 & 363113 & 2235149 & 20367 & 71818 & 375583 \\
\hline 45 a 49 & 10183338 & 4021739 & 27884 & 508067 & 2941488 & 16257 & 82079 & 445561 \\
\hline 50 a 54 & 8702799 & 3954061 & 24870 & 536360 & 2800865 & 14952 & 100761 & 524843 \\
\hline 55 a 59 & 7029864 & 3470947 & 24736 & 494787 & 2334623 & 13083 & 109399 & 556157 \\
\hline 60 a 64 & 5470630 & 2921140 & 22698 & 432577 & 1859226 & 11174 & 116144 & 565750 \\
\hline 65 a 69 & 4051068 & 2390407 & 20713 & 374868 & 1429941 & 9903 & 120945 & 557381 \\
\hline 70 a 74 & 3143844 & 2044104 & 20247 & 351574 & 1140927 & 9032 & 134599 & 555031 \\
\hline 75 a 79 & 2180488 & 1551147 & 20547 & 293046 & 791513 & 8450 & 140889 & 472118 \\
\hline 80 ou mais & 2476433 & 1990981 & 54165 & 452636 & 852329 & 22458 & 311257 & 661386 \\
\hline Rural (anos) & 29821150 & 7132347 & 67896 & 1023312 & 4510069 & 54645 & 309196 & 1258009 \\
\hline O a 4 & 2494759 & 74002 & 3368 & 4780 & 22052 & 2512 & 2274 & 11046 \\
\hline 5 a 9 & 2830886 & 193012 & 3192 & 16140 & 99867 & 3045 & 6933 & 36398 \\
\hline 10 a 14 & 3207424 & 318974 & 3054 & 28652 & 194635 & 4362 & 10239 & 47148 \\
\hline 15 а 19 & 2951135 & 304525 & 3500 & 27844 & 185202 & 4253 & 9057 & 40582 \\
\hline 15 a 17 & 1867215 & 191232 & 2106 & 17380 & 117494 & 2550 & 5650 & 26023 \\
\hline 18 e 19 & 1083920 & 113293 & 1394 & 10464 & 67708 & 1704 & 3407 & 14559 \\
\hline 20 a 24 & 2526790 & 288179 & 3647 & 26389 & 169307 & 4833 & 8490 & 41067 \\
\hline 25 a 29 & 2329702 & 303183 & 4164 & 28842 & 176850 & 4577 & 8833 & 43816 \\
\hline 30 a 34 & 2131265 & 330066 & 3792 & 32839 & 190555 & 4299 & 10777 & 49921 \\
\hline 35 а 39 & 1917595 & 375786 & 3780 & 38473 & 225012 & 4563 & 11476 & 55063 \\
\hline 40 a 44 & 1827767 & 588442 & 3903 & 75022 & 406978 & 3476 & 13718 & 69396 \\
\hline 45 a 49 & 1651309 & 741753 & 3348 & 109028 & 539586 & 2467 & 15551 & 83864 \\
\hline 50 a 54 & 1431523 & 751068 & 3314 & 118872 & 536366 & 2456 & 19197 & 100882 \\
\hline 55 a 59 & 1254569 & 699239 & 3332 & 110599 & 484944 & 2437 & 21190 & 111929 \\
\hline 60 a 64 & 1032657 & 603135 & 3157 & 95188 & 399422 & 2094 & 24877 & 121026 \\
\hline 65 а 69 & 801720 & 504287 & 3345 & 83154 & 318305 & 2022 & 26191 & 120923 \\
\hline 70 a 74 & 600893 & 407525 & 3405 & 74868 & 240818 & 1539 & 29580 & 114657 \\
\hline 75 a 79 & 390198 & 288483 & 3919 & 60297 & 155576 & 1550 & 28863 & 89147 \\
\hline 80 ou mais & 440958 & 360689 & 11676 & 92325 & 164595 & 4160 & 61950 & 121143 \\
\hline
\end{tabular}

Fonte: Instituto Brasileiro de Geografia e Estatística (IBGE). 


\section{Hipertensão arterial importante ou cardiopatia}

Atualmente, são 17 milhões de brasileiros hipertensos. Até 2025 , esse número deve crescer $80 \%$, segundo estudo conjunto da Universidade do Estado de Nova York, Escola de Economia de Londres e do Instituto Karolinska (Suécia) $)^{3}$.

\section{Diabetes mellitus tipo 1}

Segundo a International Diabetes Federation (IDF), há 13,4 milhões de diabéticos no Brasil (tipos 1 e 2). Trata-se de um número alarmante, já que a estimativa era de que haveria cerca de 12,7 milhões de brasileiros diabéticos somente em $2030^{4}$.

\section{Epilepsia}

A epilepsia é a condição neurológica grave de maior prevalência do mundo, acometendo $1 \%$ da população. Existe tratamento eficaz para a epilepsia, porém não está disponível para todos. Somente 10 a 40\% dos pacientes recebem tratamento medicamentoso 5 .

\section{Câncer controlado em acompanhamento}

São esperados 520 mil novos casos de câncer por ano no Brasil. Em 2010, foram registradas 176.098 mortes por neoplasia maligna no país ${ }^{6}$.

\section{Obesidade mórbida}

No início de fevereiro de 2013, foram divulgados os resultados de um levantamento realizado pelo Programa Meu Prato Saudável, coordenado pelo Instituto do Coração (Incor), do Hospital das Clínicas da Faculdade de Medicina da Universidade de São Paulo (USP), apontando que $66,3 \%$ dos entrevistados estão acima do peso: $28,9 \%$ estão obesos - sendo $19,0 \%$ com obesidade grau 1 (forma mais leve), 7,2\% com grau 2 , e $2,7 \%$ com o grau 3, conhecido como obesidade mórbida - e 37,4\% com sobrepeso.

\section{Insuficiência renal crônica ou hepatopatia grave}

Dados da Sociedade Brasileira de Nefrologia indicam que 10 milhões de brasileiros sofrem de alguma disfunção renal. Hoje, entre 90 e 100 mil pessoas passam por diálise no país. O Brasil, de acordo com a Organização Mundial da Saúde, é considerado um país com prevalência intermediária, situada entre 2,5 e 10\% da população.

\section{Tuberculose}

Anualmente são notificados cerca de seis milhões de novos casos em todo o mundo, levando mais de um milhão de pessoas a óbito.

No Brasil, a tuberculose é um sério problema de saúde pública, com profundas raízes sociais. A cada ano, são notificados aproximadamente $70 \mathrm{mil}$ casos novos e ocorrem 4,6 mil mortes em decorrência da doença. O país ocupa o 170 lugar entre os 22 países responsáveis por $80 \%$ do total de casos de tuberculose no mundo 7 .

\section{Síndrome da imunodeficiência adquirida}

Os números do Ministério da Saúde mostram que o Brasil está perto de registrar o caso número $600 \mathrm{mil}$ de AIDS no país. Desde o primeiro caso, em 1980, até a última atualização (junho/2010), foram contabilizados 592.914 casos de infecção pelo vírus da imunodeficiência humana (em inglês, human immunodeficiency vírus - HIV). Dentre eles, 38.538 casos foram notificados em 2009. Por ano, é registrada uma média de 36 mil novos casos. Além disso, a pesquisa do Ministério aponta que um quarto dos brasileiros portadores do HIV não sabe que está infectado. Isso corresponde a cerca de 135 mil pessoas.

\section{Espondilite anquilosante}

A incidência anual dessa doença permanece em 7,3 para cada 100 mil pessoas. Contudo, sua frequência na população em geral é estimada como sendo de 0,1 a 2,0\%. Acomete ambos os sexos, porém tem predileção pelo sexo masculino, em uma proporção de 2 a 4 homens para cada mulher, sendo, portanto, mais amena no sexo feminino. No Brasil, ocorre na proporção de 10 homens para 1 mulher ${ }^{8}$.

\section{Surdez unilateral e deficiência auditiva}

Existe uma estimativa de que, contando só no Reino Unido, sejam diagnosticados 9 mil casos de surdez unilateral anualmente. No Brasil, os números chegam a cerca de 3,6 milhões de deficientes auditivos unilaterais, ou seja, possuem perda na sensação de audição em um dos ouvidos ${ }^{9}$.

\section{Patologias psiquiátricas}

Depressão, ansiedade, Síndrome de Burnout e uso de drogas estão entre as mais frequentes. Boletim divulgado em abril de 2014, pela Previdência Social, mostra que estão entre as maiores causas de afastamento do trabalho. 


\section{DISCUSSÃO}

A lei 8.213 de 24 de julho de 1991 poderia ser considerada o marco inicial do debate sobre a inclusão de pessoas com deficiência no Brasil. Seguida de vários decretos, culminando com a Instrução Normativa 98 de 15 de agosto de 2012, que uniformizava os procedimentos adotados pela fiscalização do Ministério do Trabalho.

Os dados mostram que em 2009 foram inseridos 26.449 profissionais no mercado. Já no ano 2010, os auditores formalizaram a contratação de 28.752 pessoas com deficiência; e em 2011 esse número aumentou 19,62\%, chegando a 34.395 pessoas em todo o país ${ }^{10}$.

Este trabalho vem apresentando os dados da lei de deficiência, pois talvez seja o único mecanismo legal que ampare pessoas com restrições médicas ao trabalho, juntamente com a reabilitação profissional, que enfrenta timidamente a situação.

A legislação atual não atende às reais necessidades do Brasil e precisa ser revista.

Os afastamentos junto ao Instituto Nacional do Seguro Social (INSS) aumentam a cada ano e está grande o número de pessoas que poderiam e deveriam estar trabalhando, desde que lhes fossem oferecidas condições para isso. Essa situação traz prejuízos para todos: empresas, uma vez que os funcionários não trabalham; governo, uma vez que aumentam os custos da previdência social; e para o próprio trabalhador, que muitas vezes tem sua remuneração diminuída além de se sentir cada vez mais incapaz. Basta analisar o grande número de afastamentos por patologia psiquiátrica junto ao INSS.

O trabalhador que se apresenta para um exame médico ocupacional solicita ao médico do trabalho que verifique se ele poderá executar suas atividades adequadamente, sem colocar em risco sua integridade física ou de outros, evitando situações que causem ou agravem suas condições de saúde ou que venham a causar acidentes.

Nas áreas produtivas das empresas são comuns vários tipos de atividades que poderiam dificultar a contratação de pessoas que tenham restrições médicas. As mais comuns seriam:

- trabalho em altura;

- trabalho em espaço confinado;

- trabalho com esforços físicos;

- trabalho com uso de proteção respiratória;

- trabalho em locais com calor;
- trabalho com uso de veículos industriais;

- trabalho com ergonomia inadequada;

- trabalho em ritmo acelerado;

- trabalho com horários rígidos de descanso;

- trabalho com atendimento ao público com alta demanda emocional;

- entre vários outros tipos de trabalho que poderiam ser específicos para uma ou outra situação de restrição médica.

Professor Hudson Couto escreveu que é difícil obter um resultado satisfatório na reabilitação dos afastados principalmente porque a empresa nem sempre apresenta postos de trabalho ergonomicamente corretos em quantidade suficiente, nem sempre tem disposição para encarar o processo de reabilitação, nem sempre aceita que o trabalhador reabilitado tenha uma redução na sua cota de desempenho; e também porque após certo tempo de afastamento o trabalhador pode apresentar uma atitude negativista em relação ao retorno ao trabalho ${ }^{11}$.

Se algumas empresas relutam em readaptar seus próprios funcionários, é possível imaginar como seria se adequarem para a contratação de pessoas com restrições médicas ou até mesmo aceitarem de volta algum funcionário previamente contratado que agora apresente restrição médica.

A obra "Trabalhadores com restrições laborais - Comitê de acompanhamento em empresa pública” concluiu que a experiência multidisciplinar vivenciada pelo comitê e estendida à empresa e aos trabalhadores tem proporcionado reflexões e ampliação da compreensão do trabalho mesmo àqueles setores e trabalhadores não envolvidos diretamente. Propiciou maior interesse dos profissionais de saúde e de recursos humanos da empresa em temas essenciais como a felicidade no trabalho, os papeis da liderança na saúde do trabalhador e na sua retenção ou retorno ao trabalho, e a responsabilidade da empresa em atuar preventivamente em condições que apresentem risco potencial à saúde dos trabalhadores ${ }^{12}$.

No Brasil, que não consegue atender e tratar adequadamente seus doentes, principalmente os afastados dos grandes centros e cujo trabalho preventivo de saúde fica em segundo plano em detrimento de uma medicina resolutiva, certamente uma boa solução seria oferecer benefícios às empresas, a fim de tentar equacionar esse problema.

Jerri e Salvador citam que a saúde do trabalhador incluindo reabilitação profissional — ainda é um direito 
constitucional a ser conquistado na prática. Nas ações de atenção ao tema, o acolhimento e o reconhecimento do sofrimento presente no processo saúde-doença-trabalho podem melhorar a autoestima e a autoconfiança dos trabalhadores ${ }^{13}$.

Assim, uma opção de saída, após amplo debate com todos os agentes sociais envolvidos, seria encontrada no aumento de $1 \%$ em cada quadro existente atualmente nos critérios de deficiência e passando a aceitar que as empresas incluam nesse quadro pessoas com patologias com restrições médicas e que hoje estão desamparadas.

Para exemplificar a proposta, propõe-se a comparação a seguir.

Situação Atual:

- até 200 funcionários: $2 \%$ de pessoas com deficiência apenas;

- de 201 a 500 funcionários: $3 \%$ de pessoas com deficiência apenas;

- de 501 a 1.000 funcionários: $4 \%$ de pessoas com deficiência apenas;

- mais de 1.001 funcionários: 5\% de pessoas com deficiência apenas.

Sugestão deste trabalho:

- até 200 funcionários: $3 \%$ de pessoas com restrições médicas e deficiência;

- de 201 a 500 funcionários: 4\% de pessoas com restrições médicas e deficiência;

- de 501 a 1.000 funcionários: 5\% de pessoas com restrições médicas e deficiência;

- mais de 1.001 funcionários: $6 \%$ de pessoas com restrições médicas e deficiência.

Ademais, $50 \%$ ou mais desse efetivo deverá ser contratado obrigatoriamente pelos critérios previstos atualmente (que poderiam ser revisados, mas não é o tema deste trabalho) e os outros 50\% (ou menos, se a empresa já tiver o número de deficientes) por pessoas com as situações médicas aqui descritas.

Dessa forma, imaginando uma empresa com 1.000 funcionários que pela lei atual deve contratar 40 deficientes e muitas vezes não consegue, por sugestão deste trabalho deveria contratar 50 pessoas, sendo 25 delas, no mínimo, com deficiência pela lei atual e 25 portadoras de patologias com restrições médicas. Se essa empresa já conseguiu, por exemplo, 30 pessoas portadoras de deficiência, poderia contratar 20 outras com restrições médicas, a fim de completar seu quadro.

Por um lado, isso ajudaria a baixar um pouco o número de contratação de deficientes, sem desprotegê-los, e por outro lado abriria caminho para incluir um grupo de pessoas que hoje sofre enormemente, muitas vezes sem atendimento médico adequado, sem acesso à saúde, e que estariam amparadas de alguma forma, por meio de convênios médicos, ou pela proteção de um trabalho registrado, ou por estar sob a proteção de uma empresa, entre outros benefícios. Ajudaria também a manter no trabalho aquele trabalhador já contratado que por algum motivo passe a apresentar restrição médica.

É preciso explicitar que, uma vez incluída no cadastro da empresa, mesmo que essa pessoa melhore sua condição médica, deixando de se enquadrar no grupo (exemplo: um obeso mórbido que emagrece muito e o deixa de ser), dará a empresa o direito de mantê-lo em seu quadro, enquanto for funcionário, pois o objetivo final que seria o de melhorar ou recuperar a saúde daquele trabalhador, foi atingido.

Outro benefício a ser conseguido seria uma forte necessidade de acompanhamento da saúde dos funcionários por parte dos Programas de Controle Médico de Saúde Ocupacional (PCMSOs), que têm seu foco principal ligado às doenças ocupacionais, buscando timidamente a saúde preventiva.

O médico do trabalho deverá avaliar no momento do exame ocupacional se aquele trabalhador será capaz ou não de realizar a função para a qual está sendo avaliado, sem colocar em risco sua integridade física e de seus colegas de trabalho.

\section{NOVOS CRITÉRIOS DE INCLUSÃO SUGERIDOS}

\section{Portadores de Restrições Médicas ( $50 \%$ da nova cota)}

Existem dois pontos importantes a serem tratados inicialmente. $\mathrm{O}$ primeiro é que as pessoas nas situações médicas abordadas a seguir estejam em condições de trabalho, conforme acompanhamento médico. $\mathrm{O}$ segundo ponto são os benefícios financeiros legais que algumas das patologias abaixo descritas permitem a seus portadores usufruírem. Não é o objeto deste estudo e não há nenhuma correlação; ou seja, receber ou não esses 
benefícios não mudam em nada a condição da pessoa para efeito de contratação.

\section{Hipertensão arterial importante} com alguma complicação (diabete, obesidade, dislipidemia) ou cardiopatias

Níveis pressóricos comprovadamente sem tratamento, iguais ou acima de $170 \mathrm{mmHg}$ de pressão sistólica por valor igual ou maior a $110 \mathrm{mmHg}$ de diastólica, associados à obesidade (Índice de Massa Corporal [IMC] $\geq 30$ ) ou diabetes tipo II ou dislipidemia (colesterol sem tratamento acima de $250 \mathrm{mg}$ e/ ou triglicérides sem tratamento acima de $200 \mathrm{mg}$ ).

\section{Cardiopatias}

Quando impliquem em redução importante da condição laboral gerando limitações clínicas inclusive na vida pessoal, como a prática de atividades físicas e deambulação frequente em longas distâncias; enfim, que gerem limitações físicas.

\section{Diabetes mellitus tipo 1}

Em uso de qualquer tipo de insulina.

\section{Epilepsia}

Histórico de convulsões comprovadamente diagnosticadas e que o paciente sabidamente irá convulsionar sem uso de medicação, não importando há quanto tempo esteja sem convulsionar com a medicação.

\section{Câncer controlado ou}

\section{não em acompanhamento}

Neoplasia maligna de qualquer parte do corpo humano com resultado de exame anatomopatológico que comprove.

\section{Obesidade mórbida}

Com IMC igual ou maior a 40 .

\section{Insuficiência renal crônica ou hepatopatia grave}

Qualquer paciente que necessite diálise continuamente. Além disso, portadores de hepatite $\mathrm{B}$ ou $\mathrm{C}$ que necessitem acompanhamento especializado.

Estariam incluídos, também, os portadores de hepatopatias não virais, como cirrose e esteatose hepática grau III.

\section{Tuberculose}

Em tratamento ou acompanhamento e que já se encontre em condições de trabalho.

\section{AIDS}

Embora desde junho de 2014 tenha sido promulgada a Lei 12.984 que proíbe discriminar os portadores de HIV, muitas vezes o candidato ou trabalhador, por medo ou vergonha, nega sua condição.

\section{Espondilite anquilosante}

Doença reumatológica, degenerativa e crônica.

\section{Surdez unilateral ou deficiência auditiva}

Perda (bilateral, parcial ou total) de 31 decibéis (dB) ou mais, aferida por audiograma nas frequências de 500, $1.000,2.000$ e $3.000 \mathrm{~Hz}$.

\section{Patologias psiquiátricas}

Depressão, ansiedade e Síndrome de Burnout, comprovadamente em tratamento há mais de seis meses e que já tenha dois ou mais afastamentos junto ao INSS. No caso do uso de drogas, pelo menos uma internação em entidade especializada.

\section{CONCLUSÕES}

Diante dos números e resultados apresentados, concluise como louvável, contudo ainda insuficiente, o esforço do governo brasileiro e das empresas em solucionar a inclusão de pessoas com deficiência. No entanto, fica claro que um grande número de pessoas com restrições médicas se encontram hoje totalmente desamparadas pela legislação.

O ritmo de contratações de deficientes vem aumentando a cada ano - isso é fato - , mas os números ainda são tímidos.

Preocupa muito a legião de brasileiros que poderia ser chamada de "intermediária"; isto é, que não se enquadram como pessoas com deficiência e também não estão em totais condições de trabalho. Essas pessoas não conseguem trabalho ou passam a correr o risco de serem demitidas quando estão trabalhando, pela queda de sua produtividade.

$\mathrm{Se}$ for aceita a realidade que os governos municipais, estaduais e federal não são capazes de atender em sua totalidade 
os casos das patologias citadas neste trabalho, sugere-se, então, essa solução para todas as partes, pois as pessoas seriam beneficiadas por estarem trabalhando, recebendo seus vencimentos e amparadas em seus tratamentos médicos sob a proteção de uma empresa; o governo se beneficiaria da transferência do atendimento médico dessas pessoas para as empresas e teria uma redução de gastos em benefícios sociais, podendo atender a outras pessoas mais necessitadas; e as empresas se beneficiariam do atendimento ao requisito legal que hoje não conseguem, além da negociação de benefícios fiscais.

\section{REFERÊNCIAS}

1. Organização das Nações Unidas para a Educação a Ciência e a Cultura. [Internet] Declaração Universal dos Direitos Humanos [acesso em 2015 fev 23]. Disponível em: <http://unesdoc.unesco. org/images/0013/001394/139423por.pdf>

2. Prefeitura de São Paulo. Secretaria Municipal da Pessoa com Deficiência e Mobilidade Reduzida. [Internet] 2013 é declarado como "Ano Ibero-americano para a inclusão das pessoas com deficiência no mercado de trabalho" [acesso em 14 fev. 2015]. Disponível em:<http://www.prefeitura.sp.gov.br/cidade/secretarias/ pessoa_com_deficiencia/noticias/?p=49764>

3. Sociedade Brasileira de Hipertensão. [Internet] Brasil pode ter $80 \%$ a mais de hipertensos até 2025, diz pesquisa [acesso em 13 fev. 2015]. Disponível em: <http://www.sbh.org.br/geral/noticias.asp?id=69>

4. MTEsporte. [Internet] Dia Mundial da Diabetes: Alunos do JVC realizam "Pit Stop" em Alta Floresta [acesso em 13 fev. 2015]. Disponível em: $<$ http://www.mtesporte.com.br/noticias/id-80183/dia_mundial_do_ diabetes_alunos_do_jvc_realizam_pit_stop_em_alta_floresta>

5. Ferreira IL, Silva TP. Mortalidade por epilepsia no Brasil, 1980-2003. Ciênc Saúde Coletiva. 2009;14(1):89-94.

6. MariottiLG. [Internet]Aprendendocomaequipedavila[acessoem14fev. 2015]. Disponível em:<http://www.hefc.org.br/index.php?option=com_ k2\&view=item\&id=161:novidade\&tmpl=component\&print=1>
7. Ministério da Saúde. [Internet] Tuberculose [acesso em 14 fev. 2015]. Disponível em: <http://portalsaude.saude.gov.br/index. php?option=com_content\&view=article\&id=11045\&ltemid=674>

8. Clínica Amice. [Internet] Espondilite Anquilosante [acesso em 14 fev. 2015]. Disponível em: <http://amiceclinica.com.br/?p=368>

9. Surdez Unilateral. [Internet] Explicação do Caso [acesso em 14 fev. 2015]. Disponível em: <http://surdezunilateral.org/?page_id=24>

10. Brasil. Portal Brasil. [Internet] Regras para contratação de pessoas com deficiência são atualizadas [acesso em 14 fev. 2015]. Disponível em: <http://www.brasil.gov.br/governo/2012/08/regras-paracontratacao-de-pessoas-com-deficiencia-sao-atualizadas>

11. Couto HA, Nicoletti SJ, Lech O. Gerenciando a LER e os DORT nos tempos atuais. Belo Horizonte: Ergo; 2007.

12. Schveitzer FC. Trabalhadores com restrições laborais: comitê de acompanhamento em empresa pública. Rev Bras Med Trab. 2014;12(1):43-9.

13. Vacaro JE, Pedroso FS. Reabilitação profissional e a aposentadoria especial nas doenças ocupacionais. Rev Bras Med Trab.2013;11(2)60-5.

Endereço para correspondência: James César da Silva Jabour Arbex Rua Fortunato Auriema Turco, 33 - CEP: 12244-700 - São José dos Campos, (SP), Brasil - E-mail: arbexjames@uol.com.br 